NASA Technical Memorandum 83548

AIAA-84-0531

\title{
Computation of Viscous Flow in Curved Ducts and Comparison With Ex $x_{i}$ erimental Data
}
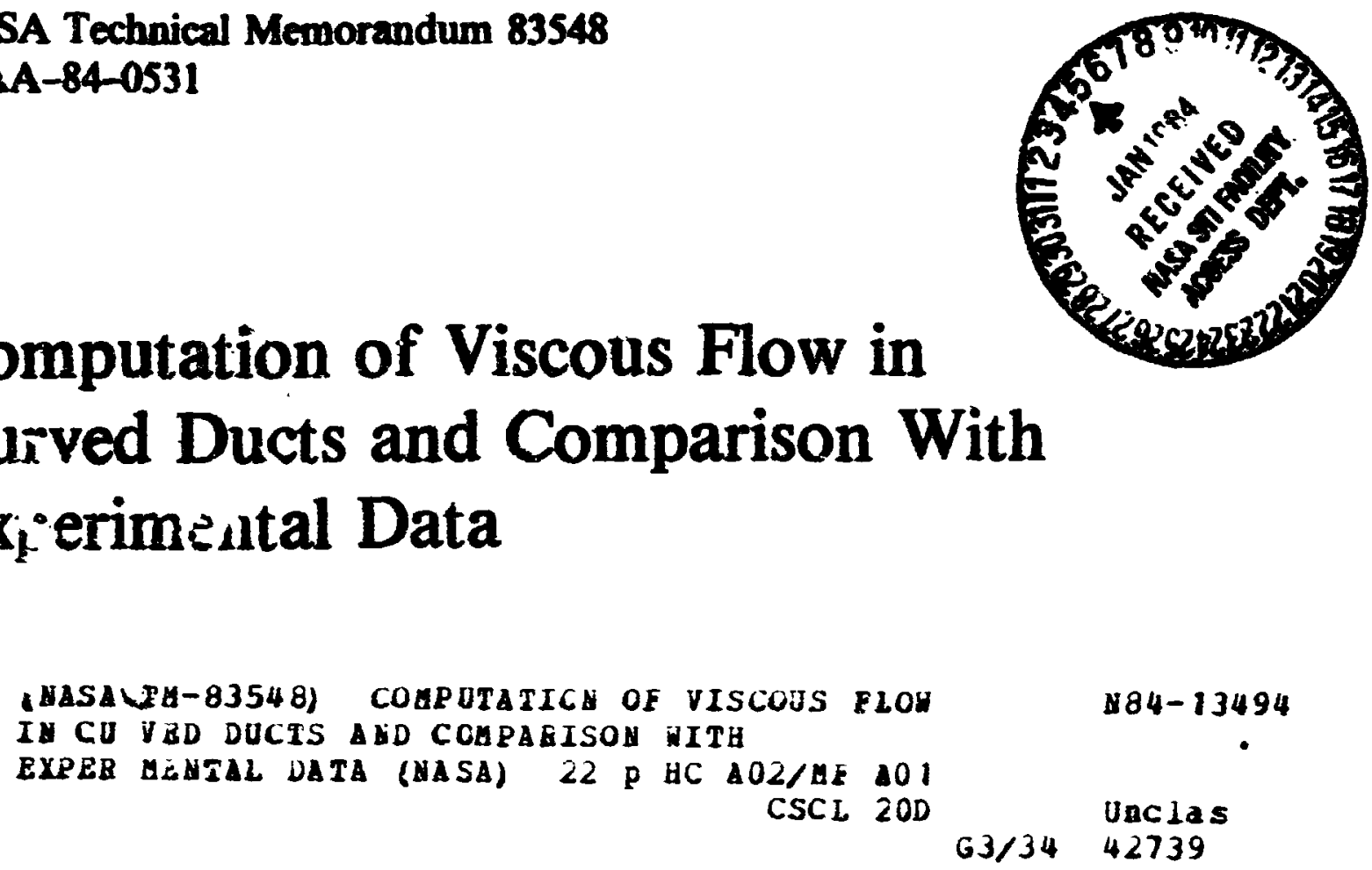

Charles E. Towne

Lewis Research Center

Cleveland, Ohio

Prepared for the

Twenty-second Aerospace Sciences Meeting sponsored by the American Institute of Aeronautics and Astronautics

Reno, Nevada. January 9-12, 1984 


\title{
COMPUTATION OF VISCOUS FLOW IN CURVED DUCTS AND COMPARISON
}

\author{
WITH EXPERIMENTAL DATA \\ Charles E. Towne \\ Naticnal Aeronautics and Space Administration \\ Lewis Researci Center \\ Cleveland, únio 44135
}

\begin{abstract}
SUMMARY
A three-dimensional analysis for fully viscous subsonic internal flow is eva'uated. The analys is, designated PEPSIG, solves an approximate form of the Navier-Stokes equations by an implicit spatial marching procedure. Resuits of calculations are presented for laminar flow through two different circular cross-sectioned 180 degree bends, and for laminar and turbulent flow through circular and square cross-sectioned 22.5-22.5 degree S-ducts. Quantitative comparisons with experimental data are shown for all cases. Special emphas is is placed on verifying the ability of the analys is to accurately predict the distorted flow fields resulting from pressure-driven secondary flows.
\end{abstract}

\section{INTRODUCTION}

The presence of three-dimensional subsonic internal flow is relatively common in many of today's airbreathing propulsion systems. Curved centerlines and changes in cross-sectional shape are often present, resulting in the generation of complex three-dimensional secondary flows and significant flow distortion. In addition, viscous effects are usualiy important, with the boundary layer thickness of ten comparable to the duct radius. These phenomena have important effects on overall propulsic i system performance.

Conventional boundary layer metinods will not work for these complex flows. A full Navier-Stokes solution could te used, but would require large amounts of computer time and storage. However, by making certain approximations to the Navier-Stokes equations, such as reglecting streamwise diffusion, a set of equations can be derived for fully viscous internal flow that can be solved by forward marching in space. An analys is method using tnese equations to compute three-dimensional subsonic flow through curved ducts with super-elliptic crosssections was developed by Briley and McDonald (ref. 1) and by Levy, McDonald, Briley, and Kreskovsky (ref. 2), and was designated PEPSIG. A preliminary evaluation of the PEPSIG method was presented by Towne and Anderson (ref. 3). However, in that evaluation orly qualitative comparisons were made with data. In addition, ieveral changes have since been made to the analysis by Levy, Briley, and Mc nonald (ref. 4).

The objective of the present study, therefore, was to evaluate and verify the most recent version of the PEPSIG analys is by making detailed quantitative comparisons between predicted results and benchmark experimental data. Special emphasis was flaced on testing the ability of the analys is to accurately predict the disturited flow field resulting from pressure-driven secondary fiows. 


\section{GOVERNING EQUATIONS}

In this analysis, the flow is computed by a single sweep spatial marching procedure which solves an approximate form of the Navier-Stokes equations. It is assumed that the flow is primarily in the direction of the duct centerline, with transverse secondary flow. This allows two basic assumptions to be made. The first is that second derivatives in the primary flow direction are negligible. The second is that the pressure in the primary, or streamwise, mpmentum equation can be represented by the sum of a known ihree-dimensional pressure field and a one-dimensional correction computed as part of the marching procedure to account for viscous blockage. When these two assumptions are applied to the Navier-Stokes equations, a set of equations can be derived that can be solved by forward marching in the primary flow direction.

The details of the derivation of the governing equations have been presented elsewhere (refs. 1, 2, and 4). For completeness, however, a brief discussion is presented here. The equations are first written in a center line-based orthogonal reference coordinate system denoted by $x_{1}, x_{2}$, and $x_{3}$, with corresponding velocities $v_{s}, w_{s}$, and $u_{p}$. The $x_{3}$ direction is the primary flow direction, and is aligned with the duct centerline at each marching step. The $x_{1}$ and $x_{2}$ directions are normal to the duct centerline and define the transverse, or secondary, flow plane. The primary momentum equation is then given by

$$
\begin{aligned}
\frac{\rho u_{p}}{h} \frac{\partial u_{p}}{\partial x_{3}}+\rho v_{s} \frac{\partial u_{p}}{\partial x_{1}}+\rho w_{s} \frac{\partial u_{p}}{\partial x_{2}} & +\frac{\rho u_{p} v_{s}}{h} \frac{\partial h}{\partial x_{1}}+\frac{\rho u_{p} w_{s}}{h} \frac{\partial h}{\partial x_{2}}=\frac{1}{h} \frac{\partial}{\partial x_{3}}\left(p_{i}+p_{v}\right) \\
& +\frac{1}{h} \frac{\partial}{\partial x_{1}}\left(h_{\mu} \frac{\partial u_{p}}{\partial x_{1}}-\mu u_{p} \frac{\partial h}{\partial x_{1}}\right)+\frac{1}{h} \frac{\partial}{\partial x_{2}}\left(h_{\mu} \frac{\partial u_{p}}{\partial x_{2}}-\mu u_{p} \frac{\partial h}{\partial x_{2}}\right) \\
& +\frac{\mu}{h} \frac{\partial h}{\partial x_{1}}-\frac{\partial u_{p}}{\partial x_{1}}-\frac{\mu u_{p}}{h^{2}}\left(\frac{\partial h}{\partial x_{1}}\right)^{2}+\frac{\mu}{h} \frac{\partial h}{\partial x_{2}} \frac{\partial u_{p}}{\partial x_{2}}-\frac{\mu u_{p}}{h^{2}}\left(\frac{\partial h}{\partial x_{2}}\right)^{2}
\end{aligned}
$$

This equation, and the others to be presented in this section,. are taken directly from reference 4 . Here $h$ is the metric scale coefficient associated with the primary direction $x_{3}$. (The metric scale coefficients for the transverse directions are both unity). Also, $p_{j}=p_{j}\left(x_{1}, x_{2}, x_{3}\right)$ is the known threedimensional pressure field and $p_{v}=p_{v}\left(x_{3}\right)$ is the unknown one-dimensional viscous blockage correction. In this study, $p_{i}$ was computed using a threedimensional potential flow analysis, thus bringing elliptic effects due to geometry into the solution.

The secondary velocities $v_{s}$ and $w_{s}$ are split into irrotational and rotational components. The continuity equation can then be writter as

$$
\frac{\partial}{\partial x_{1}}\left(\rho h \frac{\partial \varphi}{\partial x_{1}}\right)+\frac{\partial}{\partial x_{2}}\left(\rho h \frac{\partial \varphi}{\partial x_{2}}\right)+\frac{\partial}{\partial x_{3}}\left(\rho u_{p}\right)=0
$$


Here $\varphi$ is a scalar potential corresponding to the irrotational component of the secondary velocity.

The transverse momentum equations are cross-differentiated and combined to form an equation for the transport of streamwise vorticity in the primary flow direction, given by

$$
\begin{aligned}
\frac{\rho u_{p}}{h} \frac{\partial \Omega}{\partial x_{3}}+\frac{\partial}{\partial x_{1}}\left(\rho v_{s} \Omega\right) & +\frac{\partial}{\partial x_{2}}\left(\rho w_{s} \Omega\right)+\frac{\partial w_{s}}{\partial x_{3}} \frac{\partial}{\partial x_{1}}\left(\frac{\rho u_{p}}{h}\right)-\frac{\partial v_{s}}{\partial x_{3}} \frac{\partial}{\partial x_{2}}\left(\frac{\rho u_{p}}{h}\right) \\
& -\frac{\partial}{\partial x_{1}}\left(\frac{\rho u_{p}^{2}}{h} \frac{\partial h}{\partial x_{2}}\right)+\frac{\partial}{\partial x_{2}}\left(\frac{\rho u_{p}^{2}}{h} \frac{\partial h}{\partial x_{1}}\right)+\frac{\partial \rho v_{s}}{\partial x_{1}} \frac{\partial v_{s}}{\partial x_{2}}-\frac{\partial \rho v_{s}}{\partial x_{2}} \frac{\partial v_{s}}{\partial x_{1}}+\frac{\partial \rho w_{s}}{\partial x_{1}} \frac{\partial w_{s}}{\partial x_{2}} \\
& -\frac{\partial \rho w_{s}}{\partial x_{2}} \frac{\partial w_{s}}{\partial x_{1}}-\frac{\partial}{\partial x_{1}}\left[\frac{1}{h} \frac{\partial}{\partial x_{1}}(h \mu \Omega)\right]-\frac{\partial}{\partial x_{2}}\left[\frac{1}{h} \frac{\partial}{\partial x_{2}}\left(h_{\mu \Omega}\right)\right]=0
\end{aligned}
$$

where

$$
\begin{aligned}
& v_{S}=v_{\varphi}+v_{\psi}=\frac{\partial \varphi}{\partial x_{1}}+\frac{1}{\rho h} \frac{\partial h_{\psi}}{\partial x_{2}} \\
& w_{S}=w_{\varphi}+w_{\psi}=\frac{\partial \varphi}{\partial x_{2}}-\frac{1}{\rho h} \frac{\partial h_{\psi}}{\partial x_{1}}
\end{aligned}
$$

Here $\psi$ is the stream function, or vector potential, corresponding to the rotational component of the secondary velocity, and $\Omega$ is the vorticity in the $x_{3}$ direction.

The fourth equation is simply the definition of $\Omega$ written in terms of $\psi$.

$$
\frac{\partial}{\partial x_{1}}\left(\frac{1}{\rho h} \frac{\partial h_{\psi}}{\partial x_{1}}\right)+\frac{\partial}{\partial x_{2}}\left(\frac{1}{\rho h} \frac{\partial h_{\psi}}{\partial x_{2}}\right)+\Omega=0
$$

The energy equation is eliminated by assuming constant total enthalpy. For turbulent flow, an algebraic mixing length turbulence model is used.

The above equations are transformed into a non-orthogonal body-fitted coordinate system. They are then solved by forward marching in the streamwise direction from an initial station, where the flow profiles are known, by an implicit finite-difference technique. The boundary conditions that are applied result in no-slip at the walls, and symmetry at the symmetry plane. Details of the solution procedure can be found in references 1,2 , and 4 .

\section{COMPUTED RESULTS}

The results of the PEPSIG analys is have been compared with experimental data for a variety of geometric configurations and flow conditions. In this 
section, results are presented and discussed for the following cases: (1) incompressible laminar flow through two different circular cross-sectioneo 180 degree bends tested by Agrawal, Talbot, and Gong (ref. 5); (2) incompressible laminar and turbulent flow through a circular cross-sectioned 22.5-22.5 S-duct tested by Taylor, Whitelaw, and Yiannesk is (ref. 6); and (3) incompressible laminar and turbulent flow through a square cross-sectioned 22.5-22.5 S-duct also tested by Taylor, Whitelaw, and Yiannes is (ref. 7). All of these data are of benchmark quality, with streamwise and cross-flow velocities measured using laser-doppler velocimetry. PEPSIG results for other configurations are presented by Anderson (ref. 8) and by Vakili, et al. (ref. 9).

\section{Degree Bend}

The two geometries tested in reference 5 are shown in figure 1 . Both have a cross-section radius $r$ of $1.905 \mathrm{~cm}(0.75$ in.). They had centerline radii $R$ of $13.335 \mathrm{~cm}$ (5.25 in.) and $38.1 \mathrm{~cm}(15.0 \mathrm{in.})$, corresponding to radius ratios $R / r$ of 7 and 20 , respectively.

$R / r=7$. - The first results to be presented are for the $R / r=7$ bend. The flow was laminar, with a Reynolds number Re of 242 based on the crosssection radius $r$ arid the average velocity $\bar{u}$, corresponding to a Dean number De of 183. The Dean number is defined as $\mathrm{De}=2 \operatorname{Re} \sqrt{r / R}$. With a $20 \times 20$ mesh in haif the cross-section (taking advantage of symmetry), and 181 streamwise marching steps, 1.7 minutes of CPU time were needed on a Cray-1 computer.

The computed secondary flow pattern after 60 degrees of turning is shown in figure 2. Each arrow in this figure represents the direction and magnitude of the secondary velocity at the tail of the arrow. This is the classic pattern for flow in a curved pipe. The low energy flow near the wall migrates circumferentially away from the outside, or pressure side, of the bend and toward the inside, or suction side. The higher speed flow in the core region moves toward the outside of the bend due to centrifugal effects. A pair of counter-rotating vortices is thus established. This secondary flow pattern persists through the length of the duct.

The resulting flow distortion is shown in figure 3 in the form of contours of constant streamwise velocity. These results are presented at the same stations used in the experiment. The boundary layer initially thickens slightly on the outside of the bend due to the pressure increase there as the flow enters the bend. By the second station, at 30 degrees, the effect of the secondary flow is apparent in the thickening of the boundary layer on the inside of the bend. By 60 degrees of turning the secondary flow has caused significant flow distortion. A pocket of low speed flow has formed on the inside of the send, displacing the region of maximum velocity toward the outside of the bend. The results at the final two stations are similar.

In the experiment of reference 5 , data were taken at five streamwise stations. At each station, streamwise velocity profiles were measured along the five data lines shown in the inset to figure 4 . The computed streamwise velocity profiles along these data lines are compared with the data in figure 4. Computed results are shown for both $20 \times 20$ and $40 \times 40$ cross-sectional meshes; however, the effect of greater mesh resolution for this case is negligible. 
For both meshes, the agreement between the PEPSIG results and the data is excellent.

The computed and experimental secondary velocity profiles are presented in figure 5 . These results, both computed and experimental, are for slightly different flow conditions. The Reynolds and Dean numbers were 183 and 138 , respectively. The distance between adjacent data lines in figure 5 corresponds to a $v / \bar{u}$ value of 0.36 . The agreement between theory and experiment is generally very good.

$R / r=20$. - For the $R / r=20$ case, the flow was laminar with a Reynolds number of 1263 and Dean number of 565 . Several different meshes were used. For a $50 \times 50 \times 226$ mesh, 13.1 minutes of CPU time were used on a Cray-1 computer.

The computed secondary flow pattern after 84 degrees of turning is shown in figure 6 . The basic pattern is similar tc that seen in the $R / r=7$ case, but the boundary layers are thinner, the secondary velocities are not as large, and the vortices are tighter. The computed streamwise velocity contours are shown in figure 7. Again, these results are qualitatively similar to those in the $R / r=7$ case. However, the flow is more distorted, and becomes distorted after less turning.

In figures $8(a)$ and (b), the computed streamwise velocity profiles are compared with the data. (The results along data line 5 are not shown because of problems encountered in interpolating the computed results in the high velocity gradient region near the wall.) In figure $8(a)$, results for both $25 \times 25$ and $50 \times 50$ cross-sectional meshes are shown. A total of 226 points were used in the streamwise direction, with $\Delta \theta=0.5$ degrees for $\theta$ between 0 and 45 degrees, and $\Delta \theta=1.0$ degree for $\theta$ greater than 45 degrees. The agreement is generally very good for both meshes. In fact, the $25 \times 25$ mesh yives better results than the $50 \times 50$ mesh at $\theta=108$ degrees. However, the coarser mer $i$ is unable to resolve the velocity peak near the inner wall at $\theta=35$ degrees seen along data lines 2 and 3 . In figure $8(b)$, the results for the same $50 \times 50 \times 226$ mesh are shown along with results for a $50 \times 50 \times 181$ mesh with $\Delta \theta=1.0$ degree for the entire duct. With the larger streamwise step size, the analys is is again unable to resolve the inner wall velocity peak at $\theta=35$ degrees. These results indicate that proper mesh resolution in both the transverse and streamwise directions is critical in correctly predicting the initial development of the secondary flows.

Finally, in figure 9 the computed and experimental streamwise velocity contours are compared after 84 degrees of turning. The computed results are for the $50 \times 50 \times 226$ mesh. The agreement between theory and experiment is excellent.

\section{Circular S-Duct}

The circular cross-sectioned S-duct geometry tested in reference 6 consisted of two 22.5 degree circular arc bends, as shown in figure 10 . The crosssection diameter $D$ was $48 \mathrm{~mm}$ and the centerline radius $R$ was $336 \mathrm{~mm}$, for a ratio $R / D$ of 7 . 
Laminar flow. - For the laminar flow case, the Reynolds number based on cross-section diameter $D$ and average velocity $\bar{u}$ was 790 , corresponding to a Dean number of 211 . With a $20 \times 20 \times 80$ mesh, this case required 0.8 minutes of CPU time on a Cray-1 computer.

The computed secondary velocities near the inflection and exit planes are shown in figure 11 . In the first bend the pressure-driven secondary flow pattern typical of curved pipes is set up. In the second bend, the cross-flow pressure gradients change sign, tending to attenuate the secondary flows set up in the first benc. In fact, by the time the exit plane is reached, a vortextype motion has been set up in the lower half of the duct (i.e., near the inner wall of the second bend) that is in the opposite sense as that in the first bend. However, as noted in references 6,7 , and 10 , the distortion of the streamwise flow in the first bend causes the generation of streamwise vorticity in the top half of the second bend that is in the same sense as that already present, in accordance with the Squire and Winter formula for the development of streamwise vorticity. The vortices set up in the first bend are thus sustained, or even intensified, in the second bend and become concentrated near the top of the duct.

The effect of the secondary flows on the streamwise flow is shown by the streamwise velocity contours in figure 12. The boundary layer along the bottom of the duct initially thickens due to the adverse pressure gradient there as the flow enters the duct. By the time the second station is reached, just ahead of the inflection plane, the secondary flows have caused the boundary layer in the top half of the duct to thicken significantly. Through the inflection region an adverse pressure gradient exists along the top of the duct, and the analys is predicts a small region of separated flow. This separation region has no appreciable effect on the rest of the flow, and the "FLARE" approximation (ref. Il) is used to allow the streamwise marching analys is to continue. In the second half of the duct the concentration of the vortices near the top causes a severely distorted flow field. In addition, the secondary velocities near the wall in the bottom half of the Juct cause the boundary layer there to thicken. The flow at the duct exit is as distorted, or more distorted, than it would be in a unidirectional bend with the same total amount of turning.

In figure 13, the computed strearmise velocity profiles in the symmetry plane are compared with the data. Analytical results are shown for both $20 \times 20 \times 80$ and $40 \times 40 \times 80$ meshes. Both give very good agreement with the data, and the effect of greater mesh resolution is negligible.

Turbulent flow. - The Reynolds number for turbulent flow in the circular S-duct was 48,000, corresponding to a Dean number of 12,828 . With a $50 \times 50 \times 80$ mesh, this case required 4.8 minutes of CPU time on a Cray-1 computer.

The computed secondary velocities near the inflection and exit planes are shown in figure 14. The physical phenomena here are the same as in the laminar flow case. However, the boundary layers are much thinner, resulting in generally lower secondary velocities. The vortices are also somewhat more concentrated and nearer the top of the duct. The streamwise velocity contours are shown in figure 15. Again, except for the thinner boundary layers, these results are qualitatively similar to the laminar results. 
In figure 16, the computed and experimental streamwise velocity profiles in the symmetry plane are compared with the data. In contrast to the laminar case, mesh resolution for the turbulent case has a significant effect on the results. With the $50 \times 50 \times 80$ mesh, the PEPSIG results agree very well with the data. With the $25 \times 25 \times 80$ mesh, however, the thin boundary layers and secondary flow vortices are not resolved well enough for the analys is to predict the distorted flow region at the top of the duct.

\section{Square S-Duct}

The square cross-sectioned S-duct geometry of reference 7 also consisted of two 22.5 degree circular arc bends, as shown in figure 10. The cross-section width $D$ was $40 \mathrm{~mm}$ and the centerline radius $R$ was $280 \mathrm{~mm}$, for a ratio $R / D$ of 7 , the same as the circular cross-sectioned S-bend. Since the PEPSIG computer code is presently limited to super-ellipiic cross-sections, the square cross-section was represented by a super-ellipse with an exponent of 10 .

Laminar flow. - For the laminar flow case, the Reynolds number based on cross-section width $D$ and average velocity $\bar{u}$ was 790 , corresponding to a Dean number of 211 , the same as in the circular cross-sectioned laminar flow case. With a $20 \times 20 \times 111$ mesh, this case required 1.2 minutes of CPU time on a Cray-1 computer.

The computed secondary velocities near the inflection and exit planes are shown in figure 17. The important physical phenomena for this case are the same as for the circular cross-sectioned case, and the secondary flow patterns are very similar. A pair of counter-rotating vortices is set up ir, the first bend. In the second bend, these vortices become concentrated near the top of tne duct, and the near-wall secondary velocities in the lower half of the duct change direction.

The computed streamwise velocity contours are shown in figure 18 . These results are, again, very similar to those in the circular cross-sectioned S-duct. In the first bend, the boundary layer in the top half of the duct thickens because of the secondary flows. In the second bend, the concentration of the vortices near the top of the duct again causes a severely distorted flow field. Small regions of separated flow were predicted for this case along the top of the duct near the inflection plane and along the bottom of the duct near the exit plane, where adverse pressure gradients exist.

The computed and experimental streamwise velocity profiles in the symmetry plane are compared in figure 19. Both the $20 \times 20 \times 111$ and $40 \times 40 \times 111$ mesh results agree very well with the data. The $20 \times 20 \times 111$ case shows some undesirable wiggles, however, at th.e last two stations in the middle of the duct where the mesh resolution is poor.

Turbulent flow. - The Reynolds and Dean numbers for the turbulent flow case were 40,000 and 10,690 , respectively. With a $50 \times 50 \times 111$ mesh, this case required 7.7 minutes of $\mathrm{CPU}$ time on a Cray-1 computer.

The computed secondary velocities oar the inflection and exit planes are shown in figure 20. As in the circular cross-sectioned case, these results are 
basically similar to the corresponding laminar results, but with thinner boundary layers and generally lower secondary velocities. However, the vortices at the top of the duct have essentially disappeared by the time the exit plane is reached. In addition, in the second bend the streamwise velocity contours for turbulent flow, shown in figure 21, are shaped differently than for laminar flow. Near the top of the duct they have a gentle " $W$ " shape, with the low speed flow extending furthest into the core region a short distance away from the symmetry plane. In the laminar flow case, in contrast, the low speed flow extended furthest into the core region exactly in the symmetry plane. The shape of these contour lines is in agreement with that found experimentally.

In figure 22 the computed and experimental streamwise velocity profiles in the symmetry plane are compared with the data. Computed results are shown for both $25 \times 25 \times 111$ and $50 \times 50 \times 111$ meshes. The $50 \times 50 \times 111$ mesh gives slightly better agreement with the data than the $25 \times 25 \times 111$ mesh, although the differerces are small.

\section{CONCLUDING REMARKS}

For the configurations studied, the PEPSIG analys is gave quantitatively accurate results for both laminar and turbulent flow when sufficient mesh resolution was used. In particular, the analys is correctly predicted the complex physical phenomena and distorted flow fields that occur in S-shaped ducts. For the cases with thick inlet boundary layers, $20 \times 20$ cross-sectional meshes were sufficient. However, for the thin boundary layer cases a $50 \times 50$ cross-sectional mesh was generally needed for accurate results. Proper mesh resolution in both the transverse ard streamwise directions was especially important in regions where the secondary flows were initially developing.

The PEPSIG analysis is very fast compered to the alternative, a full Navier-Stokes calculation. For the results presented in this paper, the current FEPSIG computer code processed 600-700 grid points per second on the Cray-1 computer. (The analysis is approximately 10 times slower on an IBM 370/ 3033). In reference 12, a time of 0.028 seconds per grid po int per time step is reported for a three-dimensional problem solved by an efficient full NavierStokes procedure on an IBM 370/3033. Assuming 100 time steps for a converged solution, and a speed-up factor of 10 between the IBM and Cray, the NavierStokes procedure would process 3.6 grid points per second. The PEPSIG analys is is thus approximateiy 150-200 times faster, for comparable accuracy, than an efficient Navier-Stokes procedure.

\section{REFERENCES}

1. Briley, W. R., ari McDonald, H., "Analys is and Computation of Viscous Subsonic Primary and Secondary Flows," AIAA Computational Fluid Dynamics Conference, Williamsburg, VA, July 1979, pp. 74-88.

2. Levy, R., McDonald, H., Briley, W. R., and Kreskovsky, J. P., "A ThreeDimansional Turbulent Compressible Subsonic Duct Flow A.lalys is for Use with Constructed Coordinate Systems," AIAA Paper 80-1398, July 1980. 
3. Towne, Charles E., and Anderson, Bernhard H., "Numerical Simulation of Flows in Curved Diffusers with Cross-Sectional Transitioning Using a ThreeDimensional Viscous Analysis," AIAA Paper 81-0003, Jan. 1981.

4. Levy, R., Briley, W. R., and McDonald, H., "Viscous Primary/Secondary Flow Analysis for Use with Nonorthogonal Coordinate Systems," AIAA Paper 83-0556, Jan. 1983.

5. Agrawal, Y., Talbot, L., and Gong, K., "Laser Anemometer Study of Flow Development in Curved Circular Pipes," Journal of Fluid Mechanics, Vol. 85, Pt. 3, Apr. 13, 1978, pp. 497-518.

6. Taylor, A. M. K. P., Whitelaw, J. H., and Yianneskis, M., "Developing Flow in S-Shaped Ducts. II - Circular Cross-Section Duct," NASA CR-3759. To be published.

7. Taylor, A. M. K. P., Whitelaw, J. H., and Yianneskis, M., "Developing Flow in S-Shaped Ducts. I - Square Cross-Section Duct, "NASA CR-3550, May 1982 .

8. Anderson, B. H.. "Three-Dimensional Viscous Design Methodology for Advanced Technology Aircraft Supersonic Inlet Systems," AIAA Paper 84-0194, Jan. 1984.

9. 1akili, A., Wu, J. M., Bhat, M. K., Liver, P., Hingst, W. R., and Towne, C. E., "Comparison of Experimental and Computational Compressible flow in an S-Duct," AIAA Paper 84-0033, Jan. 1984.

10. Rowe, M., "Measurements and Computations of Flow in Pipe Bends," Journal of Fluid Mechanics, Vol. 43, Pt. 4, Oct. 2, 1970, pp. 771-783.

11. Reyhner, T. A., and Flugge-Lotz, I., "The Interaction of a Shock Wave with a Laminar Boundary Layer," International Journal of Non-Linear Mechanics, Vol. 3, No. 2, June 1968, pp. IT3-199.

12. Liu, N.-S., Shamroth, S. J., and McDonald, H, "Nunierical Solution of Navier-Stokes Equations for Compressible Turbulent Two/Three Dimensional Flows in the Terminal Shock Region of an Inlet/Diffuser, "NASA CR-3723, Aug. 1983. 


\section{ORTENAL PACE IS \\ OF POOR QUALITY}
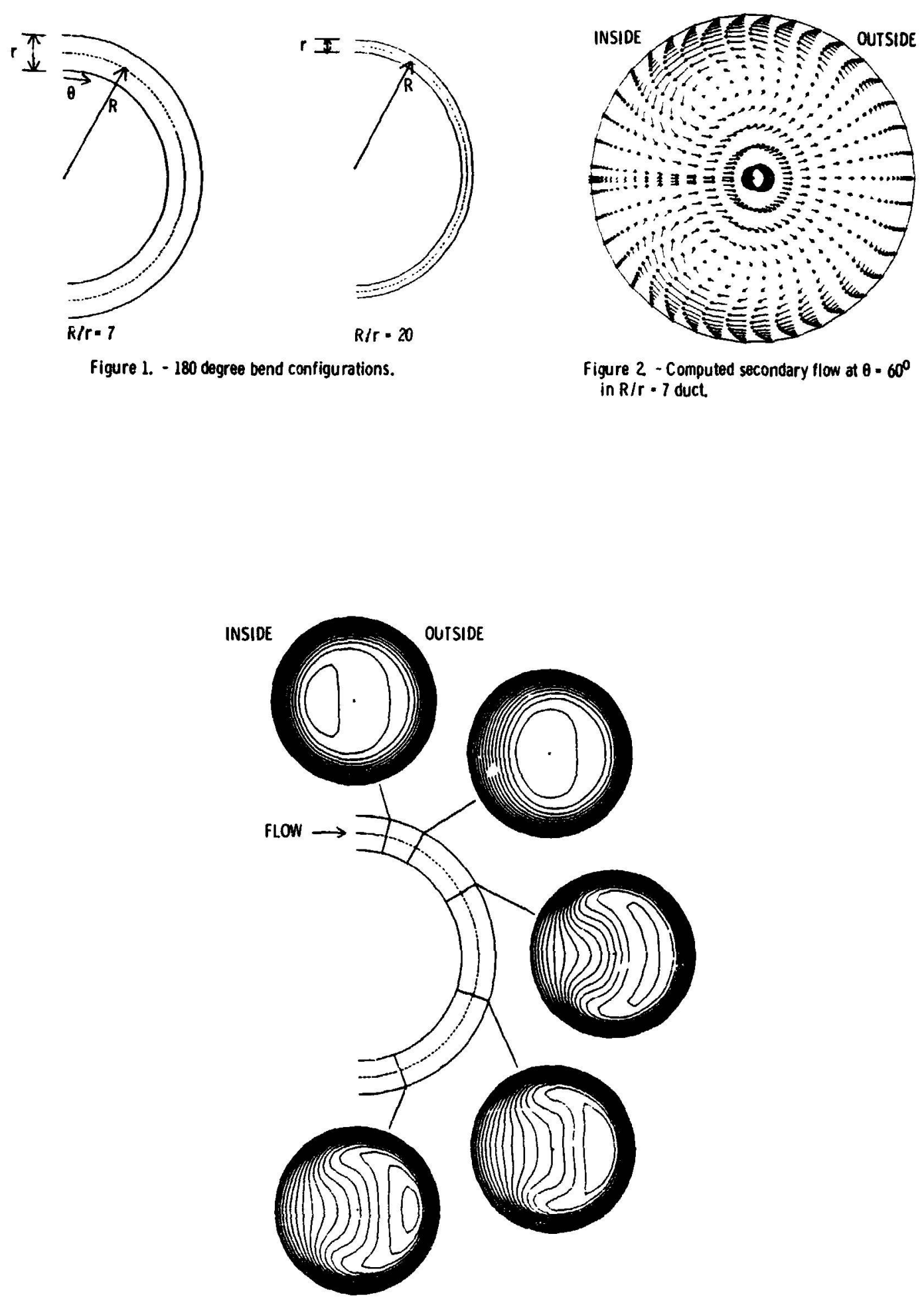

Figure 3. - Computed streamwise velocity contours in $R / T \cdot 7$ duct. 


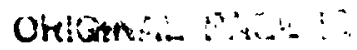
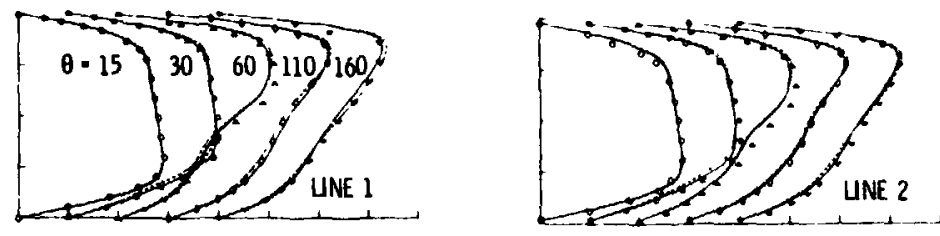

OF POOR QUALITY
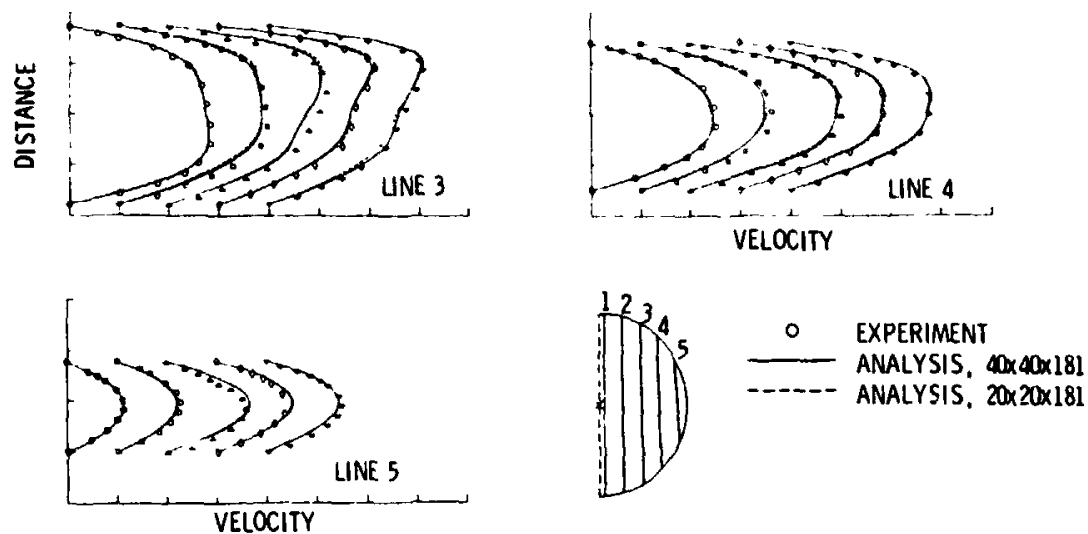

Figure 4 - Compated and experimental streamwise velocity profiles in $R / r=7$ duct

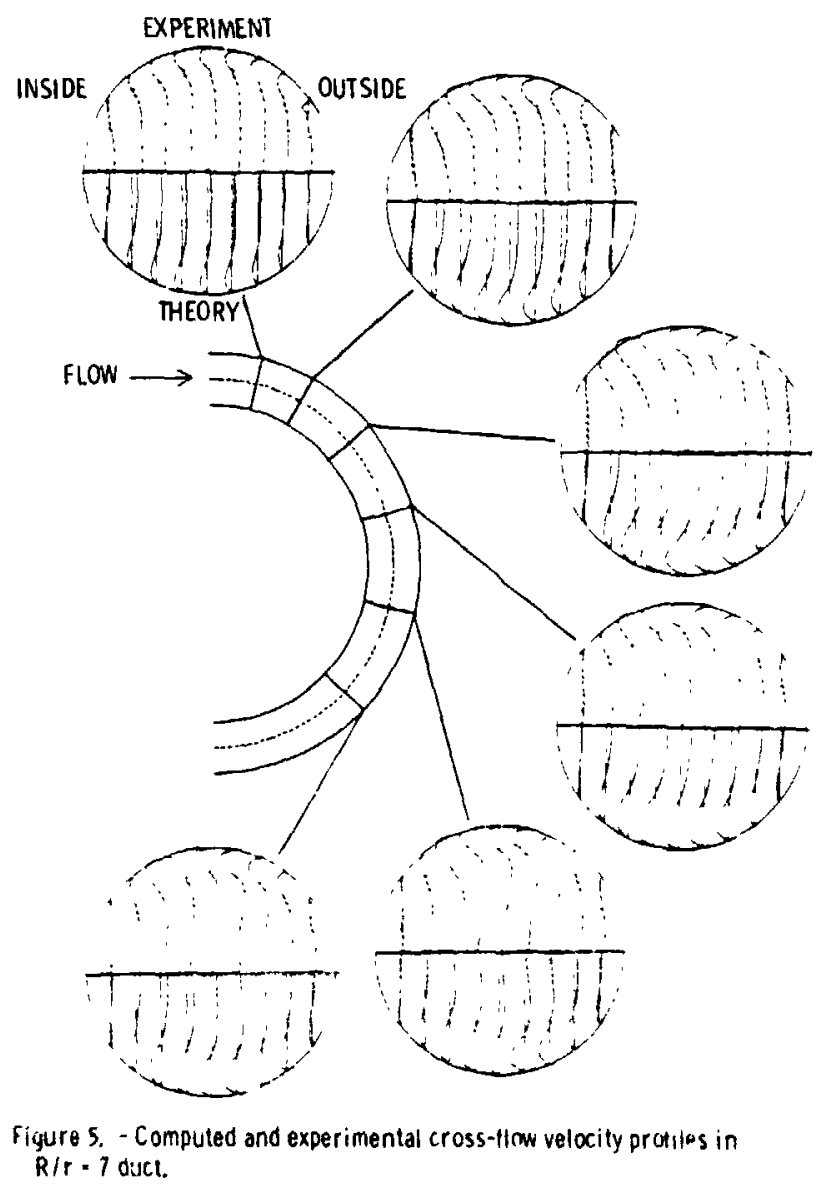




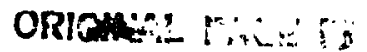

OF POOR QUALITY

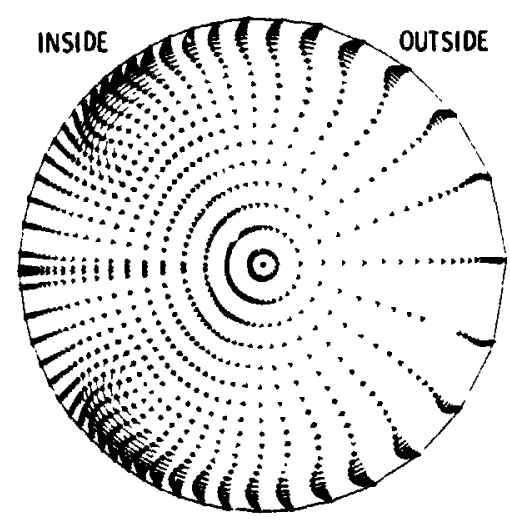

Figure 6. Computeci secondary flow at $\theta=84^{\circ}$ in $R / r=20$ duct.

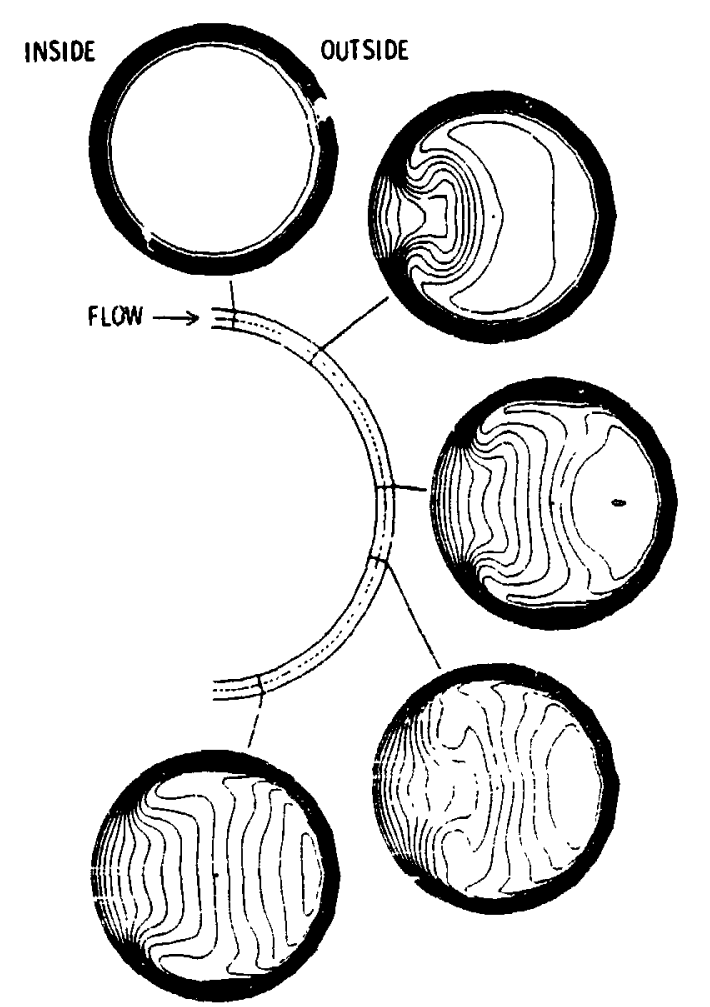

Figur 7. - Computed streamwise velccity contours in R/r $=20$ duct. 


\section{Cinixis. \\ OF POOR QUALIT}
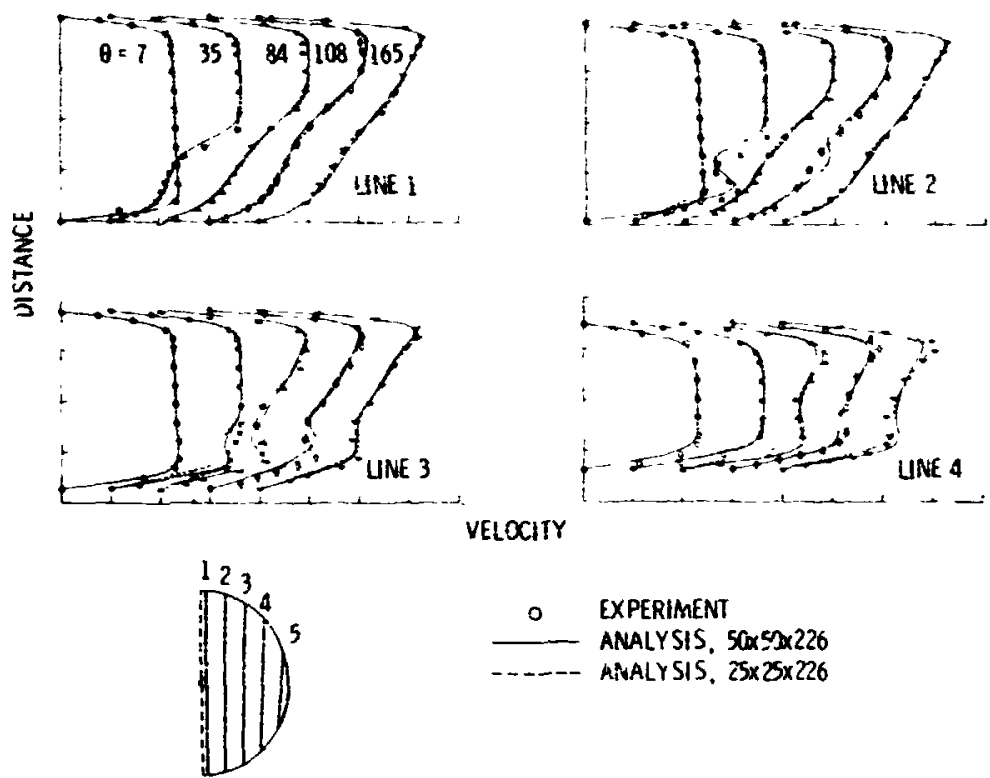

EXPERIMENT

ANALYSIS, $560 \times 5 \times 226$

ANALYSIS, $25 \times 25 \times 226$

(ai Effect of cross-sectionai mesh.

Figure \& - Computed and experimental streamwise velocity profiles in $\mathrm{RI} r=20$ duct
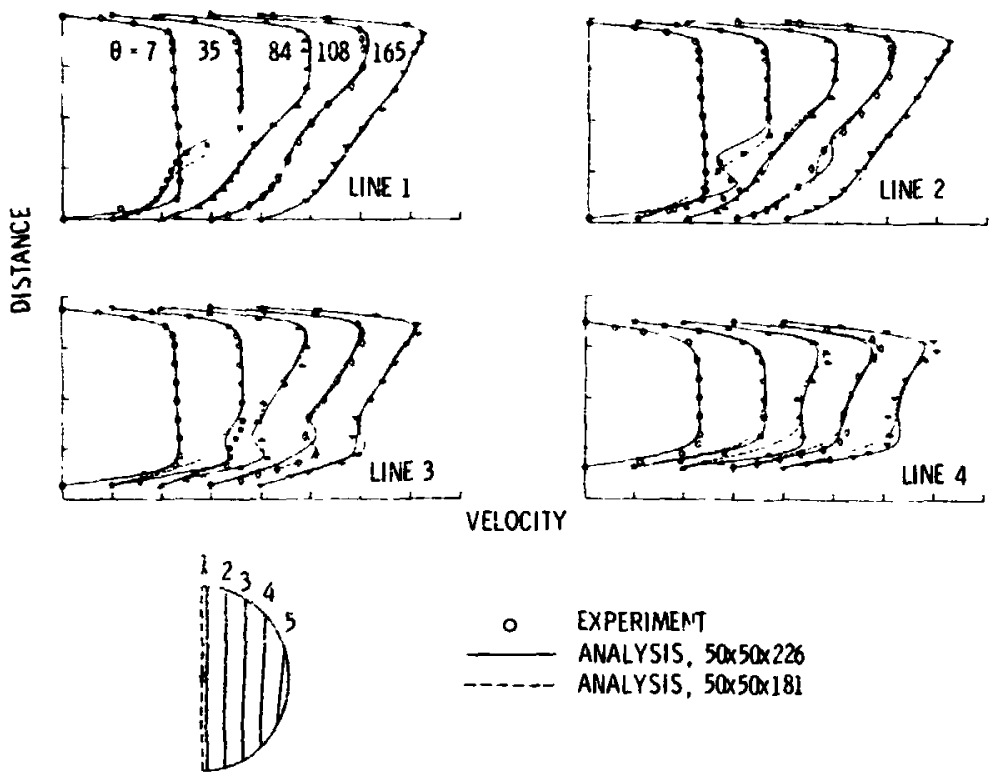

VELOCITY

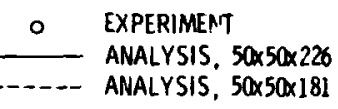

(b) Effect of streamwise mesh.

figure 8. - Concluded. 


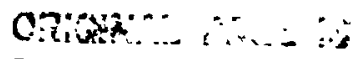

OF POOR QUALITY

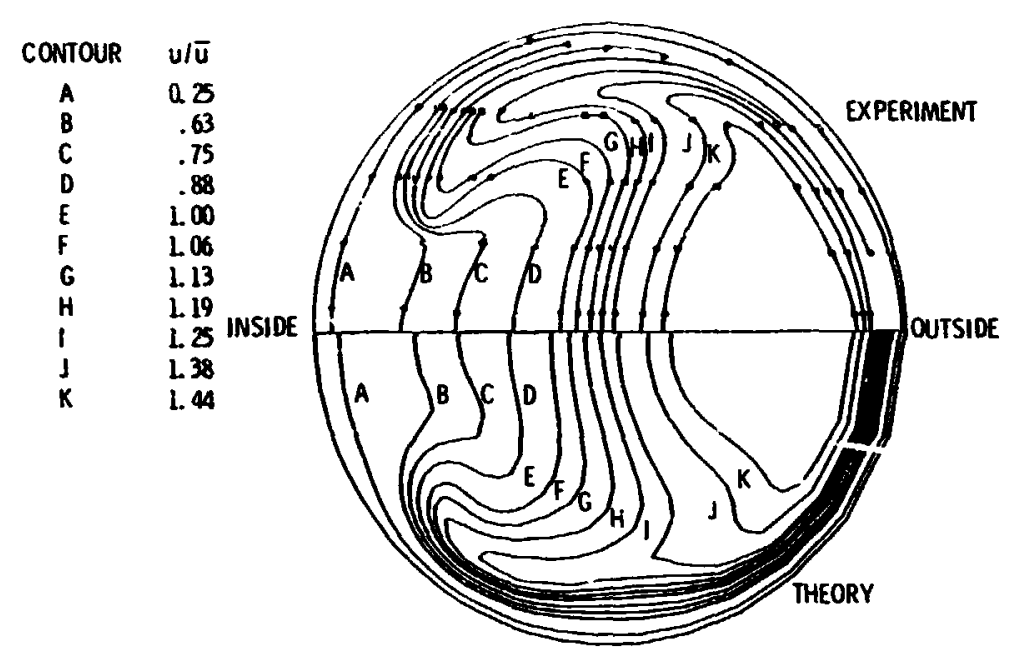

Figure 9. - Computed and experimental streamwi se velocity contours at $\theta=84^{\circ}$ in $\mathrm{R} / \mathrm{r}=20$ duct.

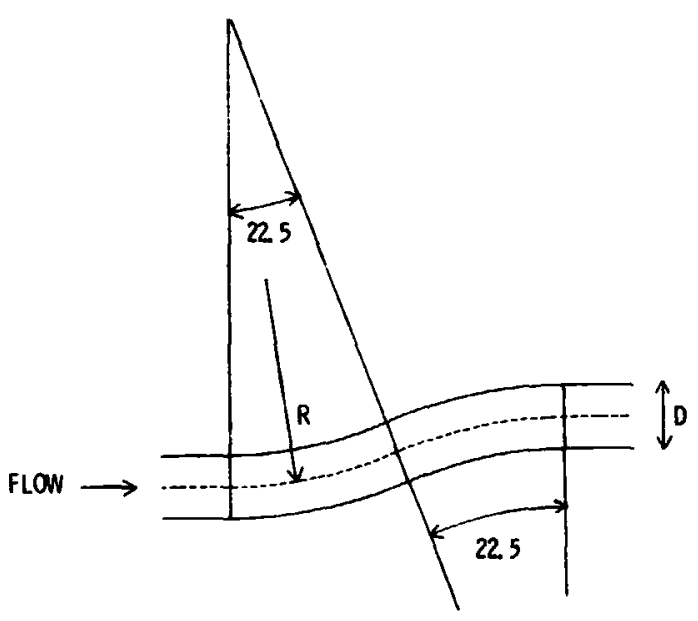

Figure 10. - S-duct configuration. 


\section{Oxisint... \\ OF POOR Quiditit}
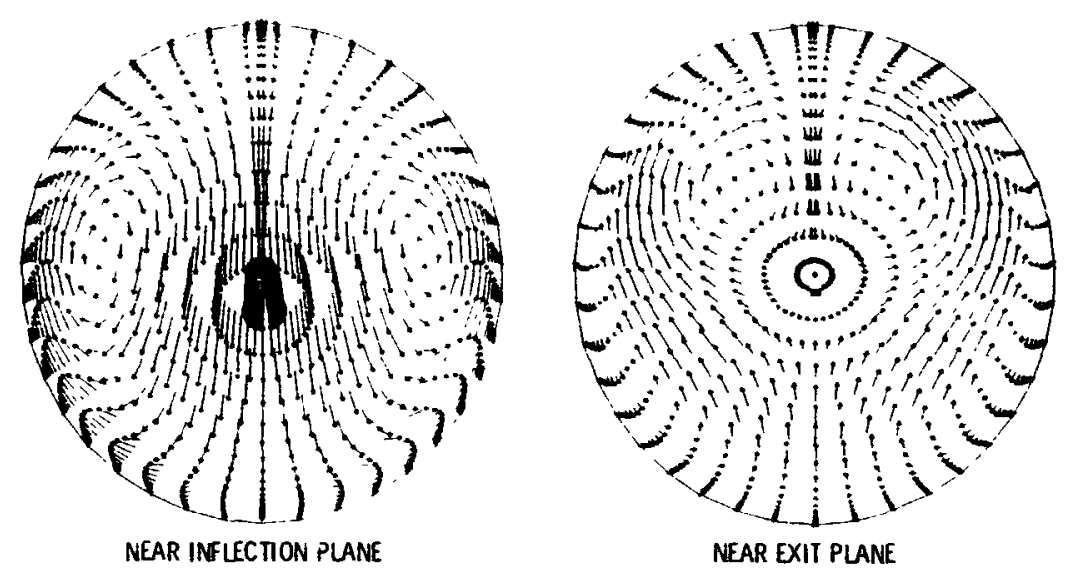

Figure 11. - Computed secondary velocities for laminar flow in 22.5-22 5 circular 5-duct.

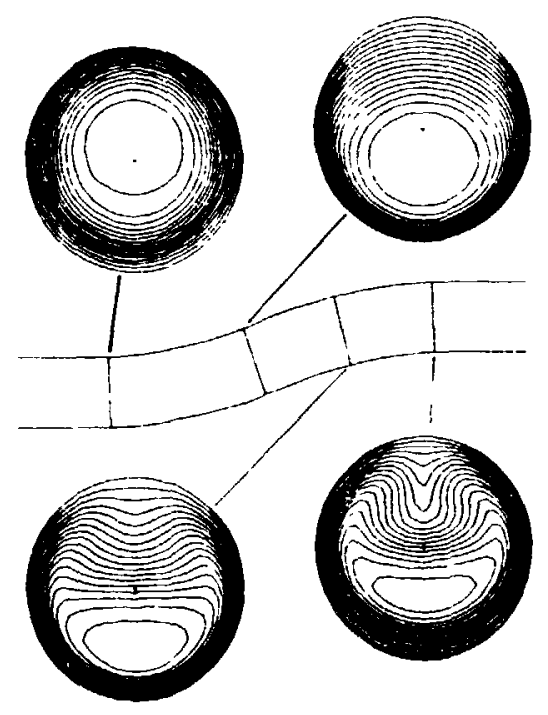

Figure 12 - Computed streamwise velocity contours for laminar flow in $22.5-22.5$ circular $\$$-duct. 


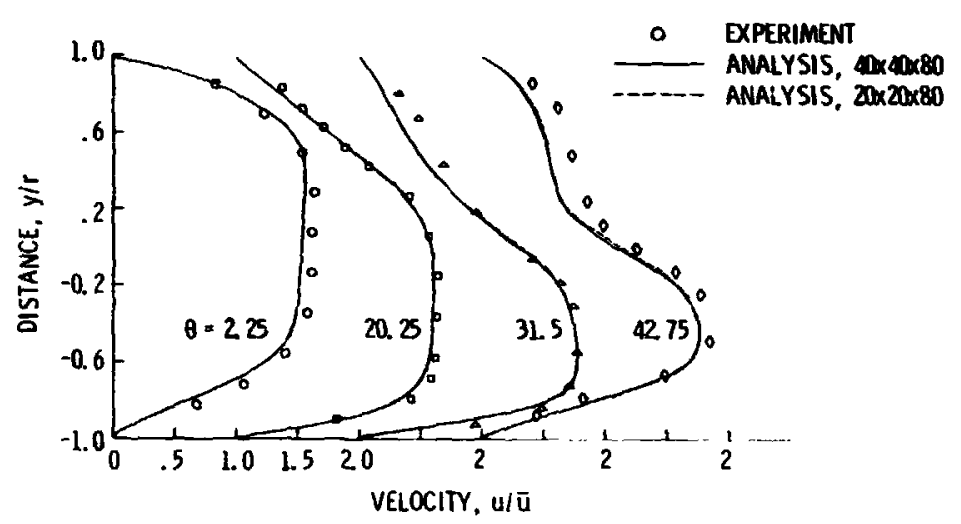

Figure 13. - Computed and experimental streanwise velocity profiles in symmetry plane for laminar flow in $225-22.5$ circular 5 -duct.
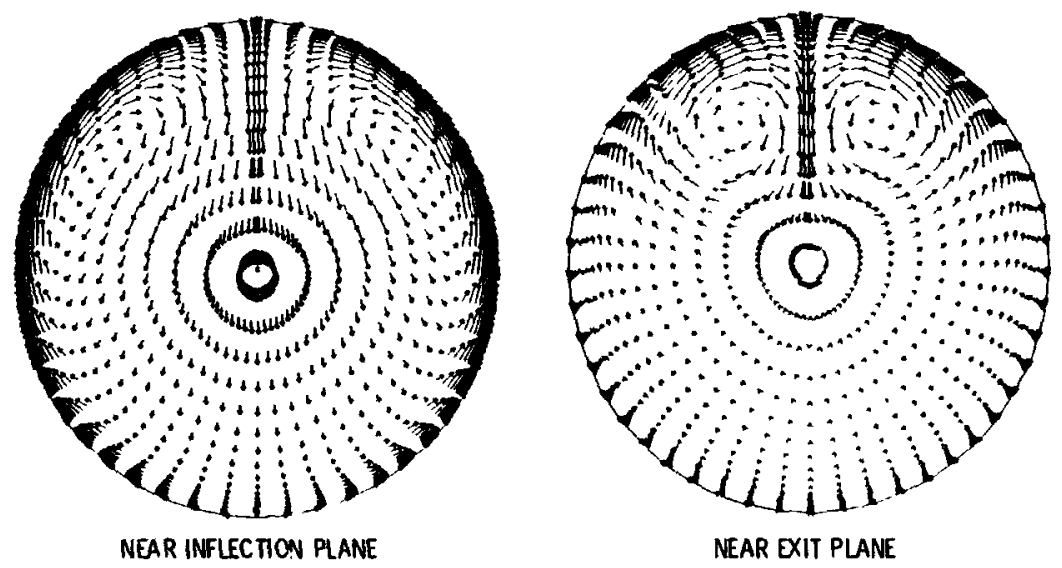

Figure 14 - Computed secondary velocities for turbulent flow in 22.5 - 22,5 circular 5 -duct. 

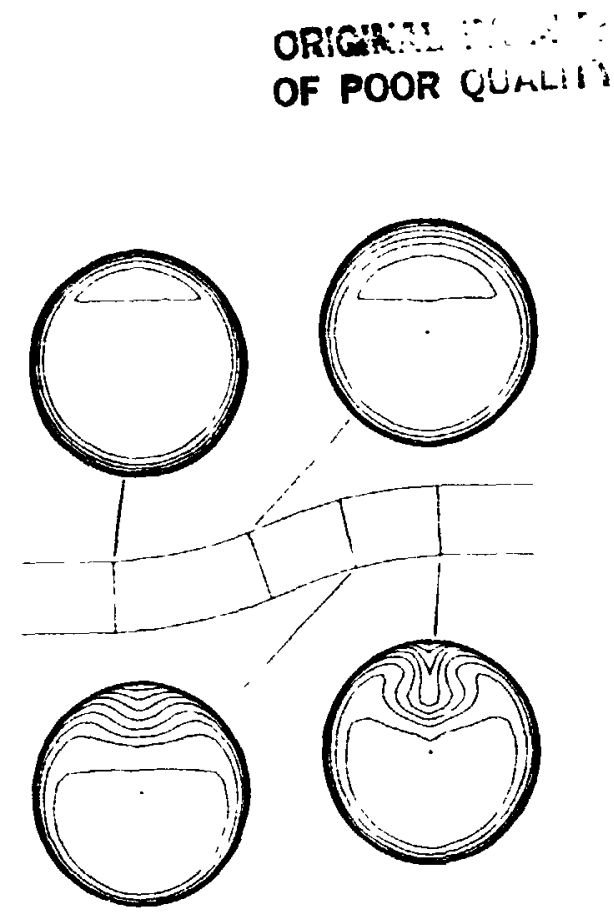

Figure 15. - Computed streamwise velocity contours for turbulent flow in 225-225 circular 5 -duct.

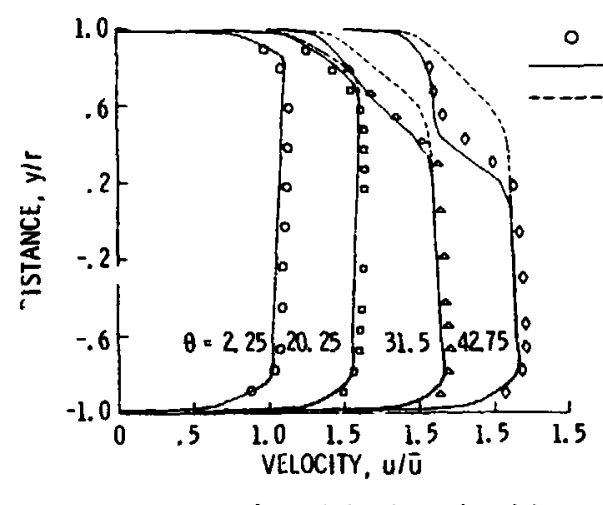

EXPERIMENT

ANALYSIS, $50 \times 50 \times 80$

ANALYSIS, $25 \times 25 \times 80$

Figure 16. - Computed and experimenta streamwise velocity profiles in symmetry plane for turbulent flow in $22.5-22.5$ circular S-duct. 
ORIGHAS I $\because \ldots:$

\section{OF POOR QUALITY}
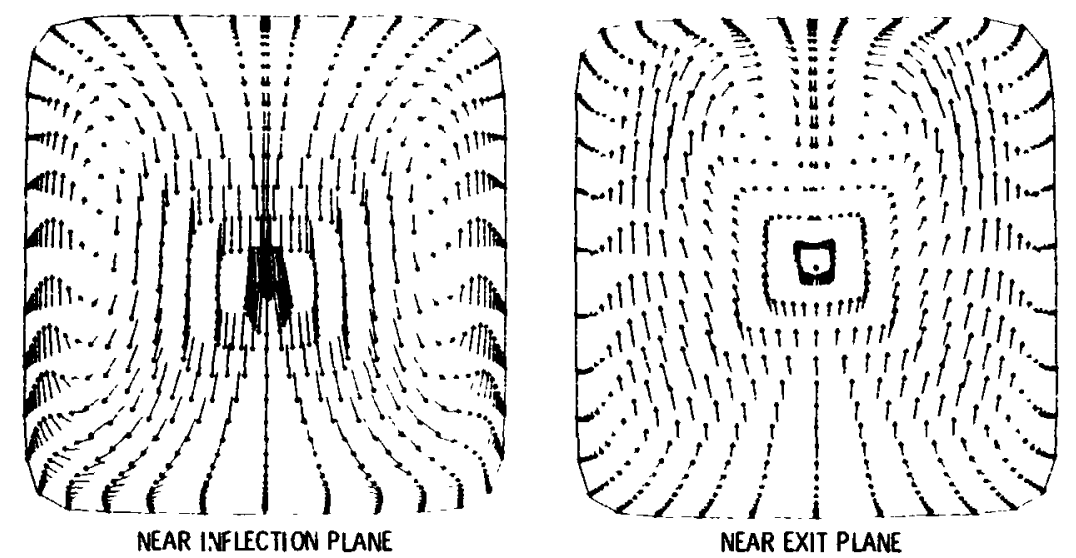

Figure 17. - Computed secondary velocities for laminar flow in $22.5-22.5$ square S-duct

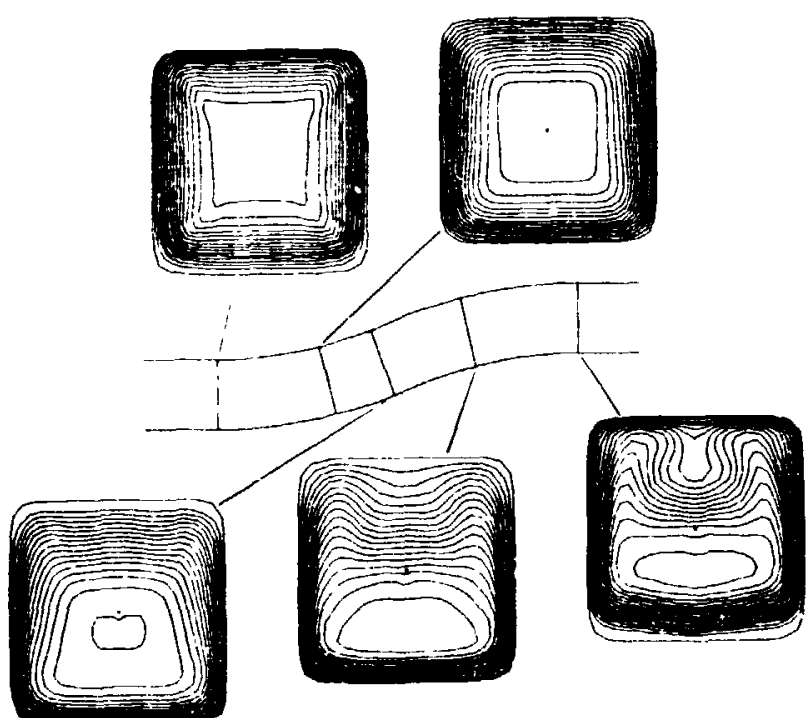

Figure 18. - Computed streamwise velocity contours for laminar flow in $225-225$ square $S$-duct. 


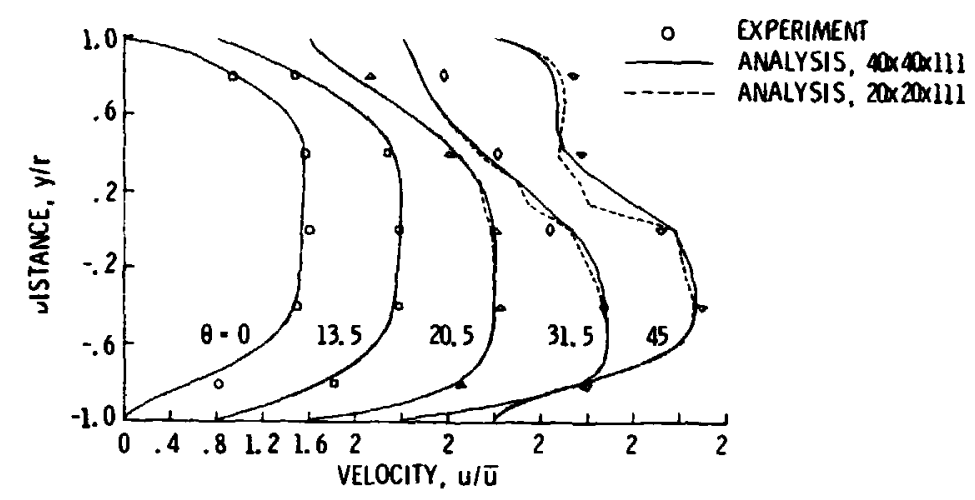

Figure 19. - Computed and experimental streamwise velocity profiles in symmetry plane for laminar flow in $225-22.5$ square S-duct.
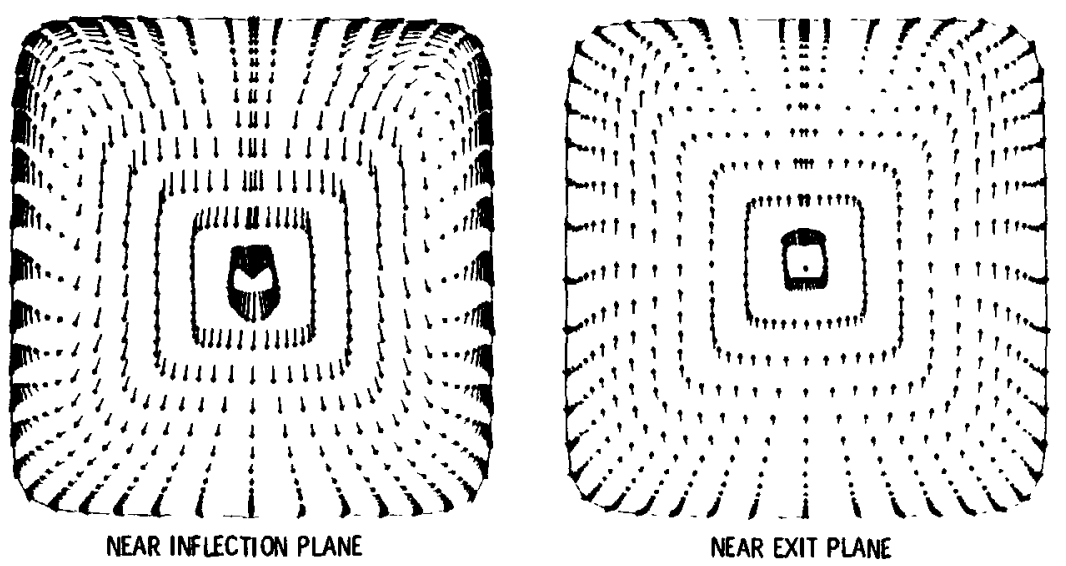

Figure 20. - Computed secondary velocities for turoulent flow in 22.5 - 225 square S-duct. 


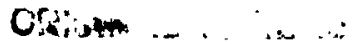 \\ OF POOF́ QUALITY}

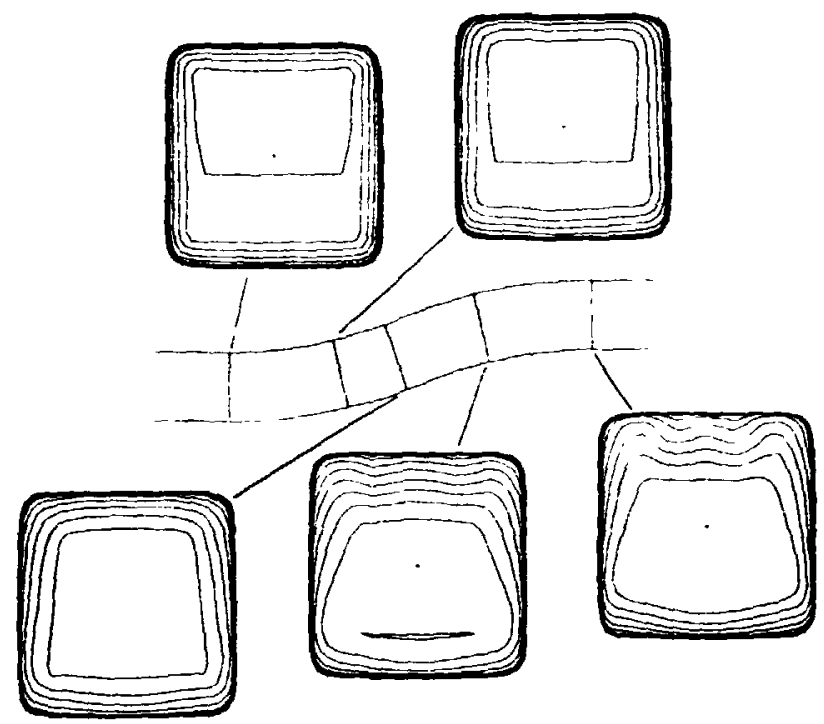

Figure 21. - Computed streamwise velocity contours for turbulent flow in 22. 5 - 22.5 square 5 -duct.

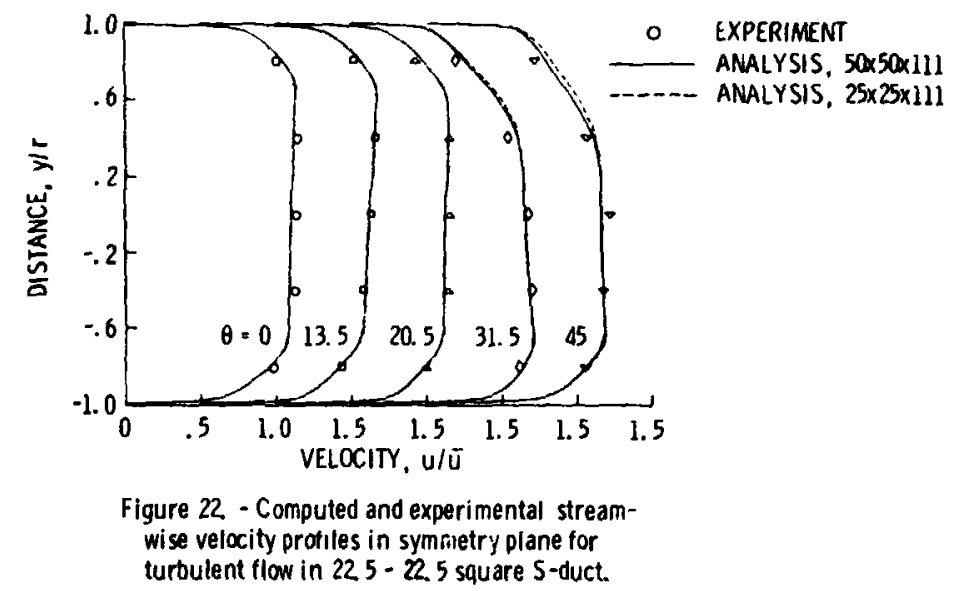

